\title{
Técnica para avaliação da pressão arterial pulmonar de equinos durante o exercício progressivo em esteira rolante ${ }^{1}$
}

\author{
Deborah P.M. Dias ${ }^{2 *}$, Luisa G. Teixeira ${ }^{3}$, Nara S. Bernardi ${ }^{2}$, Kamila Gravena ${ }^{2}$, Raquel M. \\ Albernaz ${ }^{4}$, Carlos A.A. Valadão ${ }^{2}$, Antonio de Queiroz Neto ${ }^{4}$ e José C. de Lacerda Neto ${ }^{2}$
}

\begin{abstract}
Dias D.P.M., Teixeira L.G., Bernardi N.S., Gravena K., Albernaz R.M., Valadão C.A.A., Queiroz Neto A. \& Lacerda Neto J.C. 2013. [Technique for pulmonary arterial pressure measurement in horses during the incremental exercise on a treadmill.] Técnica para avaliação da pressão arterial pulmonar de equinos durante o exercício progressivo em esteira rolante. Pesquisa Veterinária Brasileira 33(2):254-260. Departamento de Clínica e Cirurgia Veterinária, Faculdade de Ciências Agrárias e Veterinárias, Universidade Estadual Paulista, Via de Acesso Prof. Paulo Donato Castellane s/n, Jaboticabal, SP 14884-900, Brazil. E-mail: deborah_dias@hotmail.com

The aim of this study was to illustrate the surgical implantation of Swan Ganz introducers into the jugular vein, as well as the pulmonary artery cannulation procedure using ten mixed breed (MB) adult horses. We also proposed to demonstrate pulmonary arterial pressure (PAP) values measured through the implanted Swan Ganz during one incremental submaximal exercise test performed on a treadmill. Surgical implantation of the Swan Ganz introducers has demonstrated to be easy and quick to perform. None of the animals showed signs of discomfort following the surgery and ergometric test was carried out as proposed. PAP increased proportionally with exercise intensity, and the major average values were recorded at the higher effort time points. The PAP average values increased $(\mathrm{P}<0.05)$ when treadmill speed reached $7.5 \mathrm{~m} / \mathrm{s}(39 \pm 3 \mathrm{mmHg})$ and $8.5 \mathrm{~m} / \mathrm{s}(41 \pm 3 \mathrm{mmHg})$, comparing to average values at rest $(26 \pm 3 \mathrm{mmHg})$. However, the PAP values observed at the maximal effort performed by the MB horses of this trial $(41 \pm 3 \mathrm{mmHg})$, are under the values described for other breeds at similar effort tests. The technique described in the present study contributes for the standardization of research protocols using arterial pulmonary access via Swan Ganz catheter implantation in horses. The ergometric test was effective to promote PAP changes according to the effort, showing PAP values for MB horses that can guide further investigations.
\end{abstract}

INDEX TERMS: Exercise, horse, pulmonary arterial pressure, Swan Ganz, treadmill.

RESUMO.- Objetivou-se ilustrar a técnica cirúrgica de implantação de introdutores para cateter de Swan Ganz na veia jugular, bem como o procedimento de canulação da artéria pulmonar de dez equinos adultos sem raça definida (SRD). Realizando medidas através do cateter de Swan

\footnotetext{
${ }^{1}$ Recebido em 5 de setembro de 2012.

Aceito para publicação em 10 de dezembro de 2012.

${ }^{2}$ Departamento de Clínica e Cirurgia Veterinária, Faculdade de Ciências Agrárias e Veterinárias (FCAV), Universidade Estadual Paulista (Unesp), Via de Acesso Prof. Paulo Donato Castellane s/n, Jaboticabal, SP 14884900, Brasil.*Autor para correspondência: deborah_dias@hotmail.com

${ }^{3}$ Fundação Educacional Jayme de Altavila (FEJAL), Centro Universitário Cesmac, Rua Bandeirantes 249, Maceió, AL 57051-120, Brasil.

${ }^{4}$ Departamento de Morfologia e Fisiologia Animal, FCAV-Unesp, Jaboticabal, SP.
}

Ganz implantado, objetivou-se ainda demonstrar os valores de pressão arterial pulmonar (PAP) durante um teste de esforço progressivo de intensidade submáxima realizado em esteira rolante. A técnica cirúrgica de implantação de introdutores para o cateter de Swan Ganz empregada, demonstrou-se de fácil e rápida realização. Os animais não apresentaram complicações frente ao introdutor implantado e o teste ergométrico foi realizado conforme proposto. A PAP se elevou ao longo do exercício seguindo o incremento de velocidade, sendo os maiores valores médios observados nos momentos de maior intensidade do esforço. Os valores médios de PAP aumentaram $(\mathrm{p}<0,05)$ às velocidades de $7,5 \mathrm{~m} / \mathrm{s}(39 \pm 3 \mathrm{mmHg})$ e $8,5 \mathrm{~m} / \mathrm{s}(41 \pm 3$ $\mathrm{mmHg}$ ), quando comparados aos valores mensurados no repouso $(26 \pm 3 \mathrm{mmHg})$. Entretanto, os valores de PAP en- 
contrados no máximo de esforço realizado pelos equinos SRD deste ensaio $(41 \pm 3 \mathrm{mmHg})$, encontram-se abaixo dos valores descritos para outras raças para a mesma intensidade de esforço. A descrição detalhada da técnica ilustrada no presente trabalho, pode contribuir substancialmente na padronização de protocolos experimentais que propõem o acesso arterial pulmonar por meio da implantação de cateter de Swan Ganz em equinos. 0 teste ergométrico proposto foi eficaz em promover variações de PAP de acordo com o esforço realizado, demonstrando valores para equinos SRD que poderão ser usados como base para futuras avaliações.

TERMOS DE INDEXAÇÃO: Equino, esteira rolante, exercício, pressão arterial pulmonar, Swan Ganz.

\section{INTRODUÇÃO}

Grande parte do conhecimento gerado em relação às respostas fisiológicas do equino ao exercício é resultado de ensaios realizados em esteira rolante analisando alterações cardiovasculares, respiratórias, metabólicas, termorregulatórias, hematológicas e hormonais durante diferentes distâncias e intensidades de esforço (Rose \& Hodgson 1994). Considerando as avaliações cardiovasculares, o exame da perfusão pulmonar pode indicar a presença de determinadas enfermidades ou a predisposição à ocorrência das mesmas (Ducharme et al. 1999). A circulação pulmonar recebe todo o fluxo sanguíneo proveniente do ventrículo direito e perfunde os capilares alveolares, permitindo que a troca gasosa seja realizada. Já a circulação brônquica, um ramo da circulação sistêmica, recebe apenas $1 \%$ do fluxo sanguíneo do ventrículo esquerdo, irrigando as vias aéreas e outros tecidos do pulmão (Robinson 1991, Manohar et al. 1993).

0 acesso invasivo arterial pulmonar fornece informações importantes acerca da função cardiovascular de equinos atletas e em animais examinados experimentalmente, destacando-se as avaliações fisiológicas e farmacológicas, em repouso ou durante testes ergométricos. A artéria pulmonar devidamente cateterizada, utilizando-se cateter de Swan Ganz, pode ser utilizada como via para colheita de sangue venoso misto e para medidas de pressão arterial pulmonar (PAP). Para atingir a circulação pulmonar, o cateter de Swan Ganz passa pelas câmaras do lado direito do coração, após ter sido introduzido a partir de cânulas específicas implantadas na veia jugular direita ou esquerda. Milne et al. (1975) demonstraram os primeiros valores de PAP para equinos em repouso, sendo a PAP sistólica, diastólica e média respectivamente de $42 \mathrm{mmHg}, 18 \mathrm{mmHg}$ e $26 \mathrm{mmHg}$ ao nível do mar.

A ecografia cardíaca é o método mais utilizado em avaliações cardiovasculares de equinos. Entretanto, tal método não permite a avaliação funcional contínua durante o exercício (Durando 2003). Apesar de não fornecer as mesmas informações obtidas pelo estudo ecocardiográfico, a vantagem da cateterização arterial pulmonar é prover mensurações dinâmicas (em exercício) da pressão no átrio direito, da pressão arterial pulmonar e da pressão de oclusão da artéria pulmonar, sendo a última relacionada à pressão nos capilares pulmonares. A medida da pressão de oclusão é realizada mediante a insuflação do balonete, presente na extremidade do cateter de Swan Ganz, no interior da artéria pulmonar ocluindo-a. Quando a insuflação do balonete não é suficiente para ocluir a luz do vaso de forma efetiva, o valor de pressão de oclusão da artéria pulmonar pode ser obtido a partir do valor da pressão diastólica da artéria pulmonar (Bonagura \& Muir 1991).

Segundo Langsetmo et al. (1999), as pressões médias nos átrios esquerdo e direito apresentam-se marcadamente elevadas durante o exercício máximo, demonstrando valores de $70 \mathrm{mmHg}$ e $45 \mathrm{mmHg}$, respectivamente, sendo que a elevação da pressão no átrio direito aumenta diretamente a PAP e a pressão nos capilares pulmonares. 0 primeiro relato de que a PAP aumenta em equinos durante o exercício, em relação a valores de repouso, foi de Parks \& Manohar (1983). Tal incremento, que ocorre visando otimizar o influxo de sangue para os pulmões para suprir a elevada demanda de oxigênio durante o esforço, é substancialmente maior em equinos do que em outras espécies, devido ao grande volume de massa muscular recrutado para a realização do exercício (Erickson et al. 1990). Posteriormente, Magid et al. (2000) verificaram valores de PAP ( $\mathrm{mmHg}$ ) média de equinos em posição quadrupedal em repouso $(27,6 \pm 1,1)$ e, durante o exercício intenso $(107,7 \pm 4,5)$.

Estudos avaliando a PAP durante o exercício tem sido realizados buscando informações sobre a etiologia e prevenção da hemorragia pulmonar induzida pelo exercício, já que a hipertensão capilar pulmonar parece predispor à ocorrência da enfermidade (Erickson et al. 1990, Manohar et al. 1993, Mills et al. 1996, Ducharme et al. 1999, Funkquist et al. 2000, Magid et al. 2000). A avaliação de PAP em testes ergométricos tem revelado ainda sinais de alterações hemodinâmicas em equinos apresentando insuficiência mitral (Gehlen et al. 2004) e fibrilação atrial (Gehlen et al. 2006). Entretanto, a maior parte destas avaliações é realizada em equinos da raça Puro Sangue Inglês, nos quais as enfermidades cardiovasculares são mais detalhadamente observadas e estudadas. No Brasil, protocolos experimentais com equinos contam na maioria das vezes com animais sem raça definida (SRD), não havendo estudos demonstrando dados de PAP durante o exercício para estes indivíduos. Ainda, apesar de conhecida e amplamente utilizada na prática hospitalar humana, não há relatos descrevendo detalhadamente a técnica completa para a canulação arterial pulmonar em equinos. Assim, o objetivo deste ensaio foi ilustrar a técnica cirúrgica de implantação de introdutores para cateter de Swan Ganz na veia jugular, bem como o procedimento de canulação da artéria pulmonar de dez equinos adultos (SRD). Realizando medidas através do cateter de Swan Ganz implantado, objetivou-se ainda demonstrar os valores da PAP durante um teste de esforço progressivo (TEP) de intensidade submáxima realizado em esteira rolante.

\section{MATERIAL E MÉTODOS}

O protocolo experimental realizado no presente estudo está de acordo com os princípios éticos na experimentação animal adotados pelo Colégio Brasileiro de Experimentação Animal (COBEA) e foi aprovado pela Comissão de Ética e Bem Estar Animal (CEBEA) da FCAV/Unesp, Campus de Jaboticabal (Protocolo no 004302- 
06). Foram utilizados dez equinos adultos, cinco machos e cinco fêmeas, SRD, com idade média de $10 \pm 2$ anos e peso médio de $305 \pm 18 \mathrm{~kg}$. Considerados hígidos de acordo com avaliação clínica, todos os animais passaram por programas de desverminação, vacinação e controle de ectoparasitas antes de iniciar o período experimental. Os animais foram mantidos em baias individuais e receberam como alimentação $1 \%$ do peso corpóreo em alimento volumoso, constituído de feno de Tifton, $1 \%$ do peso corpóreo em ração concentrada, sal mineral e água ad libitum.

Uma semana antes do teste ergométrico, os animais foram adaptados ao exercício em esteira rolante ${ }^{5}$ durante três dias consecutivos. No primeiro dia, os animais foram conduzidos até a manta de rolagem do equipamento para acostumarem-se ao ambiente, ao piso de borracha, às barras de contenção e à cinta de segurança. Após completa acomodação com a esteira parada, o equipamento foi ligado à velocidade de 1,5 metro por segundo (m/s) durante 5 minutos (min.), para o animal acostumar-se ao movimento de passo e, desligado para acostumar-se à parada. Este procedimento foi repetido três vezes. No segundo dia, os animais foram conduzidos até a esteira e realizaram $5 \mathrm{~min}$. de passo $(1,7 \mathrm{~m} / \mathrm{s})$. A velocidade foi aumentada gradativamente até atingir $3,5 \mathrm{~m} / \mathrm{s}$, quando já trotavam, permanecendo assim por $3 \mathrm{~min}$., seguidos de mais $3 \mathrm{~min}$. a 4,5m/s e $3 \mathrm{~min}$. a 5,5m/s. Adequados ao trote alongado, a velocidade foi aumentada gradativamente até $7,5 \mathrm{~m} / \mathrm{s}$ quando os animais já deveriam galopar. A esteira foi inclinada em $5 \%$ (elevação de $5 \mathrm{~cm}$ da extremidade frontal para cada metro de comprimento da esteira), aumentando o esforço para favorecer o aprendizado do galope e, assim mantida até o animal realizar o movimento com ritmo e equilíbrio. De volta à posição horizontal, foi realizado desaquecimento compreendendo $3 \mathrm{~min}$. de trote $(3,5 \mathrm{~m} / \mathrm{s})$ seguidos de $5 \mathrm{~min}$. de passo $(1,7 \mathrm{~m} / \mathrm{s})$. No terceiro dia, a esteira foi ligada com velocidade inicial de $1,7 \mathrm{~m} / \mathrm{s}$ durante $5 \mathrm{~min}$., seguidos de $3 \mathrm{~min}$. de trote $(3,5 \mathrm{~m} / \mathrm{s})$ para aquecimento. A velocidade foi aumentada até o galope a $7,5 \mathrm{~m} / \mathrm{s}$ e mantida por $2 \mathrm{~min}$., seguidos de mais $2 \mathrm{~min}$. a 8,5m/s. 0 desaquecimento foi realizado conforme descrito anteriormente.

Para cateterização da artéria pulmonar, implantou-se na veia jugular direita um introdutor percutâneo venoso ${ }^{6}$ de 8,5 French (Fr). 0 procedimento de implantação foi realizado com o animal em posição quadrupedal, não sedado, contido por tronco e cabresto mantido por pessoa experiente. Após tricotomia e anti-sepsia rigorosas da região cervical esquerda, especialmente sobre o sulco da veia jugular, realizou-se anestesia local infiltrativa com $2 \mathrm{~mL}$ de cloridrato de lidocaína $2 \%$, sem vasoconstritor, no ponto de punção localizado no terço médio da extensão aparente do vaso. Verificada a eficácia do procedimento anestésico, iniciou-se implantação mediante punção com agulha de 18 GA $^{7}$ (Fig.1A), atra-

${ }^{5}$ Esteira Rolante Galloper, Sahinco Indústria Comércio e Representações Ltda, Palmital, SP.

${ }^{6}$ Percutaneous Sheath Introducer Set, REF SI-09875-E, Arrow International, Inc., USA.

${ }^{7}$ Introducer Needle: 18 GA x 2-1/2"(6.35cm) XTW, Arrow International, Inc., USA.

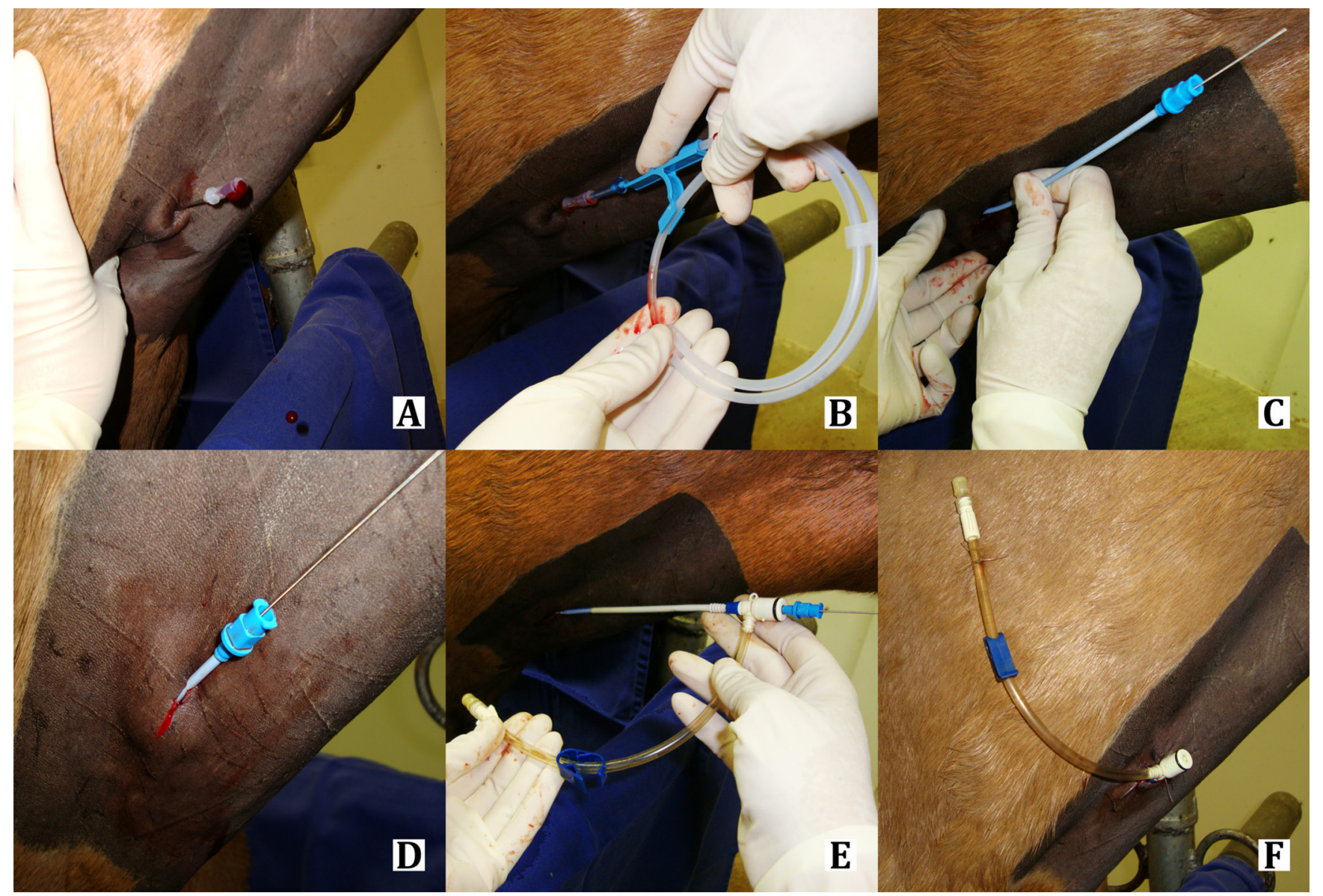

Fig.1. Diferentes etapas do procedimento cirúrgico de implantação de introdutores para cateter de Swan Ganz na veia jugular direita de equino. (A) Punção na veia jugular sobre botão anestésico; (B) Introdução do fio guia; (C) Introdução do expansor tecidual; (D) Expansor tecidual introduzido no lúmen vascular; (E) Implantação do introdutor acoplado ao extensor; (F) Aspecto ao final do procedimento. 
vés da qual introduziu-se um fio guia ${ }^{8}$ de aço de $45 \mathrm{~cm}$ (Fig.1B). Retirada a agulha, realizou-se incisão de pele de $1 \mathrm{~cm}$, paralela ao sentido da veia jugular, sobre ponto de entrada do fio guia, seguida de divulsão romba do tecido subcutâneo e muscular até identificação da parede da veia jugular. A identificação foi facilitada dilatando-se o vaso por meio de oclusão do fluxo por pressão digital em posição caudal ao ponto de punção. Um expansor tecidual ${ }^{9}$ foi introduzido na veia jugular, passado externamente ao fio guia, de modo a ampliar o orifício na parede do vaso (Fig.C,D). Esta manobra foi realizada mantendo-se a dilatação venosa por oclusão distal. 0 expansor foi então retirado e acoplado por dentro do introdutor percutâneo ${ }^{10}$ previamente preenchido com solução heparinizada estéril (10 UI de heparina sódica por mL de solução de cloreto de sódio a 0,9\%) e vedado com um adaptador PRN, para que este pudesse ser implantado na veia jugular igualmente passados externamente ao fio guia (Fig.1E). Finalmente, o introdutor foi implantado no lúmen vascular, o expansor removido, seguido de completa exteriorização do fio guia, restando somente o introdutor no lúmen da veia jugular. Realizou-se dermorrafia com um ponto em U-horizontal, utilizando-se fio de nylon monofilamento $\mathrm{n}^{\circ} 1$, sendo que este mesmo ponto foi utilizado para fixar o introdutor à pele. A fixação da extensão do cateter foi realizada com um ponto simples na pele, utilizando-se fio de nylon monofilamento no 1 (Fig.1F).

Os animais foram conduzidos até a esteira e, imediatamente antes da realização do TEP, já sobre a manta de rolagem, a artéria pulmonar foi acessada utilizando-se cateter de Swan Ganz ${ }^{11}$. A via proximal do cateter de Swan Ganz foi preenchida com solução heparinizada estéril e vedada pela seringa de $3 \mathrm{~mL}$ utilizada para injetar a solução. A via distal do cateter de Swan Ganz foi conectada a transdutor de pressão por meio de tubo extensor de $2,4 \mathrm{~m}$ de comprimento preenchido com solução heparinizada estéril. 0 transdutor foi fixado sobre uma barra estável da esteira, na altura da articulação escápulo-umeral de cada cavalo, considerando-se a relação da articulação com a altura do átrio direito. Posteriormente, o transdutor foi calibrado de acordo com a pressão atmosférica. A via elétrica do cateter foi conectada ao módulo de débito cardíaco do monitor multiparamétrico ${ }^{12}$ que registrou dados de PAP. Conectado ao monitor, iniciou-se a introdução do cateter de Swan Ganz, sob nova preparação estéril. Após percorrer o trajeto na veia jugular, a localização exata do cateter foi verificada pelas ondas e valores de pressão característicos de cada câmara cardíaca e da artéria pulmonar. Ao verificar-se a onda característica do átrio direito (Fig.2), que representa a pressão venosa central, o balonete presente na extremidade do cateter foi insuflado com 1,5 $\mathrm{mL}$ de ar, de modo a auxiliar a progressão do cateter orientado pelo fluxo sanguíneo. Seguiu-se a implantação, identificando-se a onda do ventrículo direito (Fig.3) e, finalmente da artéria pulmonar (Fig.4), quando o balonete foi desinsuflado.

A duração total de cada TEP foi de 80 min., incluindo $45 \mathrm{~min}$. de exercício, rigorosamente cronometrado segundo protocolo descrito no Quadro 1. Após $5 \mathrm{~min}$. de repouso já registrando a $\mathrm{PAP}$, iniciou-se o exercício por fase de aquecimento que incluiu 10 min. de passo $(1,7 \mathrm{~m} / \mathrm{s})$ e $5 \mathrm{~min}$. de trote $(3,5 \mathrm{~m} / \mathrm{s})$. 0 exercício progressivo foi iniciado com trote alongado à velocidade de $4,5 \mathrm{~m} / \mathrm{s} \mathrm{e}$,

\footnotetext{
${ }^{8}$ Spring-Wire Guide, Marked: 0.035" $(0.89 \mathrm{~mm})$ dia x 17-13/16" $(45 \mathrm{~cm})$. Arrow International, Inc., USA.

${ }^{9}$ Obturator: 8 Fr., Arrow International Inc., USA.

${ }^{10}$ Sheath: 8.5 Fr. X4" $(10 \mathrm{~cm})$ Arrow-Flex®, Arrow International Inc., USA.

${ }^{11}$ Thermodilution Catheter with AMC THROMBOSHIELD, REF: 131HF7, Edwards Lifesciences LLC, USA.

${ }^{12}$ Central de monitorização fisiológica DX-2010, Dixtal Biomédica Indústria e Comércio Ltda., São Paulo, SP.
}

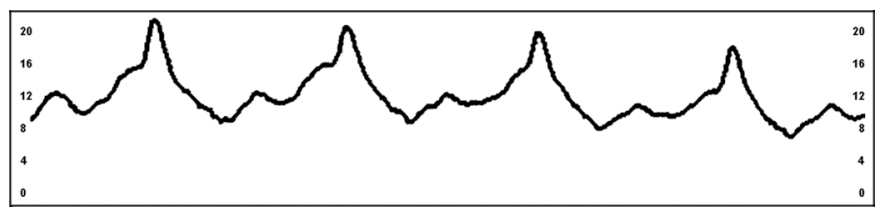

Fig.2. Aspecto da onda pressórica do átrio direito de equino obtida via cateter de Swan Ganz (Escala em mmHg).

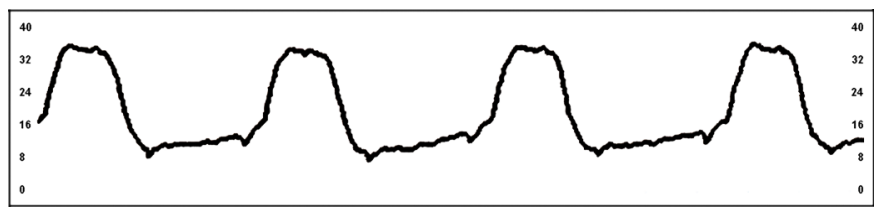

Fig.3. Aspecto da onda pressórica do ventrículo direito de equino obtida via cateter de Swan Ganz (Escala em mmHg).

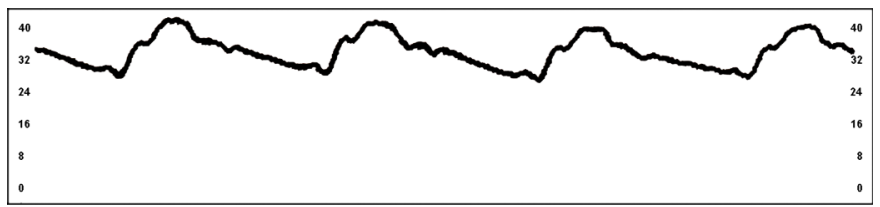

Fig.4. Aspecto da onda pressórica da artéria pulmonar de equino obtida via cateter de Swan Ganz (Escala em mmHg).

avançou em $1 \mathrm{~m} / \mathrm{s}$ a cada $3 \mathrm{~min}$. até $8,5 \mathrm{~m} / \mathrm{s}$ (galope). Finalmente os cavalos realizaram fase de desaquecimento, que incluiu $5 \mathrm{~min}$. de trote $(3,5 \mathrm{~m} / \mathrm{s})$ e $10 \mathrm{~min}$. de passo $(1,7 \mathrm{~m} / \mathrm{s})$, seguida de fase de recuperação de $30 \mathrm{~min}$. em repouso. 0 TEP proposto foi considerado de intensidade moderada ou submáximo.

Os registros de PAP foram realizados em repouso antes do exercício (M0), em movimento em nove momentos sequenciais (M1 a M9) e novamente em repouso 15 e $30 \mathrm{~min}$. após o término do exercício (M10 e M11). Durante o exercício, os valores foram tomados 30 segundos antes de cada mudança de velocidade. Acompanhando os mesmos momentos de avaliação da PAP, a frequência cardíaca (FC) em batimentos por minuto (bpm) foi registrada telemetricamente por frequencímetro ${ }^{13}$. Imediatamente após o término das avaliações, o cateter de Swan Ganz foi retirado com o animal ainda sobre a esteira. 0 introdutor permaneceu implantado na veia jugular direita por 72 horas, sendo irrigado criteriosamente de 8 em 8 horas com solução heparinizada estéril.

${ }^{13} \mathrm{~S}_{810 \mathrm{i}^{\mathrm{TM}}}$ Heart Rate Monitor, Polar Electro Ou, Kempele, Finland.

Quadro 1. Protocolo do teste de esforço progressivo realizado pelos equinos em esteira rolante e descrição dos momentos de avaliação da frequência cardíaca e pressão arterial pulmonar

\begin{tabular}{lccccc}
\hline \multicolumn{1}{c}{ Fases } & Duração & Velocidade & $\begin{array}{c}\text { Momentos } \\
\text { de avaliação }\end{array}$ & $\begin{array}{c}\text { Tempo } \\
\text { cumulativo }\end{array}$ \\
\hline Repouso & $5^{\prime}$ & $0 \mathrm{~m} / \mathrm{s}$ & M0 & $4^{\prime}$ & $5^{\prime}$ \\
Aquecimento & $10^{\prime}$ & $1.7 \mathrm{~m} / \mathrm{s}$ & M1 & $14^{\prime} 30^{\prime \prime}$ & $15^{\prime}$ \\
\multirow{3}{*}{ Exercício progressivo } & $5^{\prime}$ & $3.5 \mathrm{~m} / \mathrm{s}$ & M2 & $19^{\prime} 30^{\prime \prime}$ & $20^{\prime}$ \\
& $3^{\prime}$ & $4.5 \mathrm{~m} / \mathrm{s}$ & M3 & $22^{\prime} 30^{\prime \prime}$ & $23^{\prime}$ \\
& $3^{\prime}$ & $5.5 \mathrm{~m} / \mathrm{s}$ & M4 & $25^{\prime} 30^{\prime \prime}$ & $26^{\prime}$ \\
& $3^{\prime}$ & $6.5 \mathrm{~m} / \mathrm{s}$ & M5 & $28^{\prime} 30^{\prime \prime}$ & $29^{\prime}$ \\
Desaquecimento & $3^{\prime}$ & $7.5 \mathrm{~m} / \mathrm{s}$ & M6 & $31^{\prime} 30^{\prime \prime}$ & $32^{\prime}$ \\
& $3^{\prime}$ & $8.5 \mathrm{~m} / \mathrm{s}$ & M7 & $34^{\prime} 30^{\prime \prime}$ & $35^{\prime}$ \\
Repouso & $5^{\prime}$ & $3.5 \mathrm{~m} / \mathrm{s}$ & M8 & $39^{\prime} 30^{\prime \prime}$ & $40^{\prime}$ \\
& $10^{\prime}$ & $1.7 \mathrm{~m} / \mathrm{s}$ & M9 & $49^{\prime} 30^{\prime \prime}$ & $50^{\prime}$ \\
& $30^{\prime}$ & $0 \mathrm{~m} / \mathrm{s}$ & M10 & $65^{\prime}$ & $65^{\prime}$ \\
& $30^{\prime}$ & $0 \mathrm{~m} / \mathrm{s}$ & M11 & $80^{\prime}$ & $80^{\prime}$
\end{tabular}


Após este período, foi retirado por tração, seguida de hemostasia por compressão. Foram realizados curativos diários das feridas mediante limpeza com iodo-povidine aquoso e aplicação de pomada repelente ${ }^{14}$ por 7 dias.

Utilizando-se o software Sigma-Stat 3.0.1, os dados obtidos foram submetidos a teste normalidade (Komogorov) e análise univariada de medidas repetidas (One way ANOVA). Constatada a significância, aplicou-se o teste Tukey $(\mathrm{p}<0,05)$ para comparação das médias. Os dados apresentam-se como média \pm erro padrão da média (EPM).

\section{RESULTADOS E DISCUSSÃO}

A técnica cirúrgica de implantação do introdutor para o cateter de Swan Ganz empregada, se demonstrou de fácil e rápida realização, não ocorrendo complicações intra-operatórias. A anestesia local infiltrativa realizada, mesmo na ausência de sedação, foi eficaz em permitir que o procedimento fosse executado sem dor para o animal e com segurança. 0 tempo cirúrgico variou entre 5 e $25 \mathrm{~min}$., sendo esta variação atribuída à experiência adquirida pelo cirurgião ao longo do estudo. Por permanecer no lúmen vascular, o introdutor para Swan Ganz é pouco rígido, sendo impossível sua implantação sem o expansor tecidual. Assim, o último sempre deve ser acoplado ao introdutor para lhe conferir rigidez no momento da implantação. Os animais não apresentaram sinais de desconforto ou infecção frente ao introdutor, que permaneceu no lúmen vascular por 72 horas, como previamente determinado. Apesar de utilizado imediatamente após implantado, os introdutores foram mantidos por mais tempo visando avaliar sua viabilidade em períodos prolongados, fornecendo informações para o uso em avaliações experimentais sequenciais. Os introdutores foram retirados por tração e hemostasia por compressão, não ocorrendo sinais de hemorragia. Demonstrou-se assim que a abertura causada no vaso, apesar de maior que a de uma simples punção, não necessita de sutura para reparar a veia jugular. As pequenas feridas cicatrizaram por segunda intenção em 5 a 7 dias, não ocorrendo infecções pós-operatórias.

A implantação do cateter de Swan Ganz se deu sem maiores dificuldades, mesmo tendo sido realizada com o animal posicionado sobre a manta de rolagem da esteira. A localização do cateter foi facilmente identificada pelas ondas pressóricas obtidas, ilustradas no presente estudo como características para equinos SRD. Em alguns casos, após a verificação da onda de átrio direito e insuflação do balonete, os traçados de ventrículo e artéria pulmonar não foram observados com a progressão do cateter, supondo-se que o filamento possa ter se enovelado dentro das câmaras cardíacas. Tal complicação foi facilmente contornada desinsuflando-se o balonete, retirando-se o cateter e iniciando-se nova introdução. 0 comprimento do cateter é graduado de $10 \mathrm{em} 10 \mathrm{~cm}$ e, como os animais apresentavam dimensões semelhantes, foi possível verificar medidas próximas durante a introdução. Considerando-se que o introdutor foi posicionado no terço médio da veia jugular direita, atingiu-se o átrio direito com aproximadamente $45 \mathrm{~cm}$, o ventrícu-

\footnotetext{
${ }^{14}$ Ungüento Plus ${ }^{\circledR}$, Eurofarma Laboratórios Ltda, Rio de Janeiro, RJ.
}

lo direito com aproximadamente $55 \mathrm{~cm}$ e a artéria pulmonar com aproximadamente $85 \mathrm{~cm}$ do comprimento do cateter.

Os testes ergométricos de esforço progressivo foram realizados sem complicações e todos os registros de PAP foram obtidos conforme proposto. 0 débito cardíaco não foi avaliado, entretanto a temperatura do animal pôde ser verificada ao longo do exercício visto que o termistor presente na extremidade do cateter de Swan Ganz fornece tais informações. Considera-se de grande valia a obtenção da temperatura durante testes ergométricos, uma vez que na realização de avaliação hemogasométrica, o Swan Ganz permite a obtenção da temperatura do animal no momento da colheita de sangue para cálculo de variáveis como $\mathrm{pH}$, $\mathrm{PO}_{2}$ e $\mathrm{PCO}_{2}$. Assim, para o equino implantado com cateter de Swan Ganz acoplado ao aparelho adequado, o exercício não necessita ser interrompido para aferição de temperatura via retal. Um exemplo de comprometimento de dados experimentais importantes é o trabalho de Watanabe et al. (2006) que demonstraram valores de $\mathrm{pH}, \mathrm{PO}_{2}$ e $\mathrm{PCO}_{2}$ para equinos durante o exercício. Os próprios autores admitem que em virtude da dificuldade do acompanhamento da temperatura sanguínea durante o teste, os dados não foram corrigidos de acordo com a temperatura, subestimando os valores obtidos.

Apresenta-se a variação da FC (bpm) na Figura 5, que representa os valores obtidos no TEP realizado. Os dados de FC foram utilizados para determinação da intensidade do esforço. Considerando-se que segundo Sirotsky et al. (2010), a FC máxima ( $\mathrm{FC}_{\max }$ ) para equinos SRD é de 216,8 bpm, o teste ergométrico realizado atingiu aproximadamente a intensidade de $75 \%$ da $\mathrm{FC}_{\text {max }}$, caracterizando o exercício como submáximo. 0 trabalho de Sirotsky et al. (2010) foi a única demonstração de valores da FC durante o exercício para equinos SRD do Brasil encontrada na literatura, considerando-se assim a importância de se expor os valores obtidos no presente estudo. A média \pm EPM da FC (bpm) de M0 a M11 foi de $37 \pm 4,67 \pm 2,90 \pm 3,109 \pm 4,127 \pm 4$, $140 \pm 4,147 \pm 3,157 \pm 4,108 \pm 1,79 \pm 3,46 \pm 4$ e $43 \pm 3$, sendo os maiores valores observados nos momentos de maior intensidade do esforço. Houve aumento $(p<0,05)$ nos valores de FC de M1 a M9, quando comparados com M0. Sabe-se que incrementos de FC no exercício se devem inicialmente à

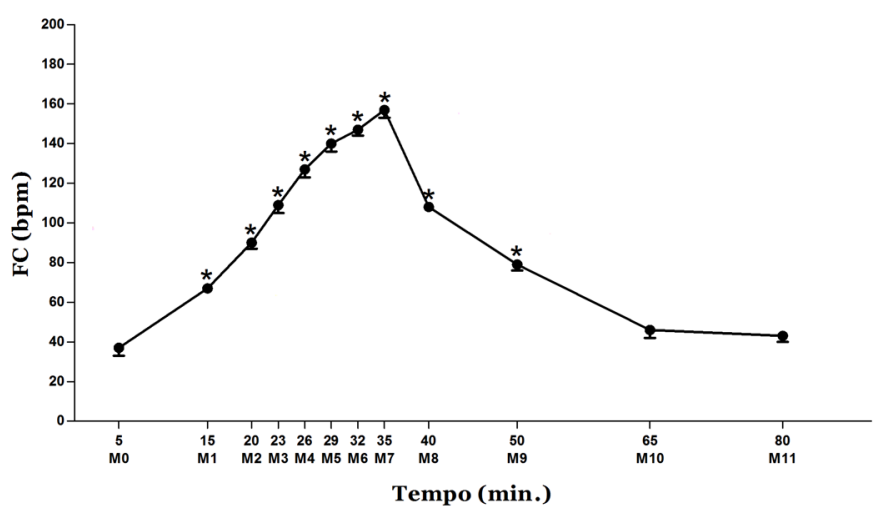

Fig.5. Variação dos valores médios \pm EPM da frequência cardíaca (bpm) no tempo em minutos (min.) de equinos em repouso (M0, M10 e M11) e durante o exercício em esteira rolante (M1-M9).

*Indicam diferenças $(\mathrm{p}<0,05)$ em relação a M0. 
inibição do tono vagal, que controla o coração no repouso, seguida da ativação simpática e liberação de catecolaminas na circulação sanguínea, gerando efeito cronotrópico positivo sobre o coração. Com o término do exercício, a estimulação simpática e a liberação de catecolaminas diminuem e o sistema nervoso autônomo parassimpático predomina novamente (Derman \& Noakes 1994, Durando et al. 2002, Poole \& Erickson 2004). Portanto, o comportamento da FC alterando-se simultaneamente com a intensidade do esforço devido à modulação autonômica está de acordo com o esperado.

A média \pm EPM de PAP (mmHg) de M0 a M11 foram de $26 \pm 3,32 \pm 2,34 \pm 3,35 \pm 3,37 \pm 3,38 \pm 3,39 \pm 3,41 \pm 3,34 \pm 2$, $30 \pm 3,27 \pm 3$ e $27 \pm 2$, estando representadas na Figura 6 . A PAP se elevou ao longo do exercício seguindo o incremento de velocidade, sendo os maiores valores médios observados nos momentos de maior intensidade do esforço. Houve aumento $(p<0,05)$ nos valores de PAP em M6 e M7, quando comparados com M0. Os valores de PAP observados no repouso para equinos SRD, corroboram os valores encontrados por Hackett et al. (2003) para Standardbreds $(28 \pm 3,9 \mathrm{mmHg})$ e cavalos Puro Sangue $(26 \pm 2,7 \mathrm{mmHg})$, os valores apresentados por Magid et al. (2000) para cavalos Puro Sangue Inglês $(27,6 \pm 1,1 \mathrm{mmHg})$, os valores obtidos por Langsetmo et al. (2000) para cavalos Puro Sangue Inglês $(29,8 \pm 0,7 \mathrm{mmHg})$ e, corroboram ainda os valores demonstrados por Muylle et al. (1984) para cavalos trotadores $(26,8 \pm 4,3 \mathrm{mmHg})$. Entretanto, os valores de PAP mensurados em M7 (41 $\pm 3 \mathrm{mmHg})$, no máximo de esforço realizado (75\% da $\mathrm{FC}_{\max }$ ) pelos equinos SRD deste ensaio, encontram-se abaixo dos valores descritos por Hackett et al. (2003) para Standardbreds $(59 \pm 6,8 \mathrm{mmHg}$ ) e cavalos Puro Sangue Inglês $(57 \pm 1,4 \mathrm{mmHg})$, à semelhante intensidade de esforço. Funkquist et al. (2000), verificaram valores médios de PAP de $72 \pm 10 \mathrm{mmHg}$ para Standardbreds durante o exercício em esteira à velocidade de $6,8 \pm 0,3 \mathrm{~m} / \mathrm{s}$, diferindo dos valores encontrados no presente estudo. Apesar de comparar exercícios de mesma intensidade, não se pode afirmar que as diferenças entre as raças são aplicáveis, pois os equipamentos utilizados para as medidas de PAP diferem entre os estudos. Assim, sugere-se a necessidade de um estudo

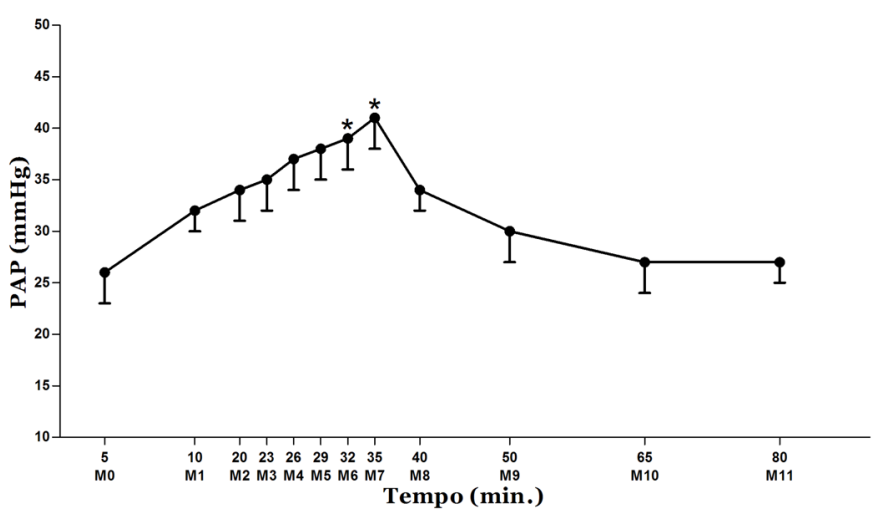

Fig.6. Variação dos valores médios \pm EPM da pressão arterial pulmonar (mmHg) no tempo em minutos (min.) de equinos em repouso (M0, M10 e M11) e durante o exercício em esteira rolante (M1-M9).

*Indicam diferenças $(\mathrm{p}<0,05)$ em relação a M0. biométrico comparando diferentes raças de equinos não somente para descrever valores de PAP, mas ainda traçar um perfil hemodinâmico completo no repouso e durante diferentes protocolos de exercício em esteira, devido à escassez de informações publicadas.

\section{CONCLUSÕES}

A descrição detalhada da técnica cirúrgica para implantação de introdutores para cateteres de Swan Ganz, bem como do procedimento de canulação da artéria pulmonar ilustrados no presente trabalho, podem contribuir substancialmente na padronização de protocolos experimentais que propõem o acesso arterial pulmonar em equinos.

0 teste ergométrico proposto foi eficaz em promover variações de PAP de acordo com o esforço realizado, demonstrando valores para equinos SRD que poderão ser usados como base para futuras avaliações.

Agradecimentos.- À Fundação de Apoio à Pesquisa do Estado de São Paulo (FAPESP) pelo apoio financeiro e institucional.

\section{REFERÊNCIAS}

Bonagura J.D. \& Muir W.W. 1991. The cardiovascular system, p.39-104. In: Muir W.W. \& Hubbell J.A.E. (Eds), Equine Anesthesia: Monitoring and emergency therapy. $2^{\text {nd }}$ ed. Mosby, St Louis.

Derman K.D. \& Noakes T.D. 1994. Comparative aspects in exercise physiology, p.15-25. In: Rose R.J. \& Hodgson D.R. (Eds), The Athletic Horse. W.B. Saunders, Philadelphia.

Ducharme N.G., Hackett R.P., Gleed R.D., Ainsworth D.M., Erb H.N., Mitchell L.M. \& Soderholm L.V. 1999. Pulmonary capillary pressure in horses undergoing alteration of pleural pressure by imposition of various upper airway resistive loads. Equine Vet. J. 30(Suppl.):27-33.

Durando M.M. 2003. Clinical techniques for diagnosing cardiovascular abnormalities in performance horses. Clin. Tech. Equine Pract. 2:266277.

Durando M.M., Reef V.B. \& Birks E.K. 2002. Right ventricular pressure dynamics during exercise: relationship to stress echocardiography. Equine Vet. J. 34(Suppl.):472-477.

Erickson B.K., Erickson H.H. \& Coffman J.R. 1990. Pulmonary artery, aortic and oesophageal pressure changes during high intensity treadmill exercise in the horse: a possible relationship to exercise-induced pulmonary haemorrhage. Equine Vet. J. 9:47-52.

Funkquist P., Nyman G. \& Persson S.G.B. 2000. Haemodynamic response to exercise in Standardbred trotters with red cell hypervolaemia. Equine Vet. J. 32:426-431.

Gehlen H., Bubeck K., Rohn K. \& Stadler P. 2006. Pulmonary artery wedge pressure during treadmill exercise in warmblood horses with atrial fibrillation. Res. Vet. Sci. 81:134-139.

Gehlen H., Bubeck K. \& Stadler P. 2004. Pulmonary artery wedge pressure measurement in healthy warmblood horses and in warmblood horses with mitral valve insufficiencies of various degrees during standardised treadmill exercise. Res. Vet. Sci. 77:257-264.

Hackett R.P., Ducharme N.G., Gleed R.D., Mitchell L.M., Soderholm L.V., Erickson B.K. \& Erb H.N. 2003. Do Thoroughbred and Standardbred horses have similar increases in pulmonary vascular pressures during exertion? Can. J. Vet. Res. 67:291-296.

Langsetmo I., Weigle G.E., Erickson H.H. \& Fedde M.R. 1999. Influence of frusemide on dynamic cardiac variables during exercise. Equine Vet. J. 30(Suppl.):170-173.

Langsetmo I., Fedde M.R., Meyer T.S. \& Erickson H.H. 2000. Relationship of pulmonary arterial pressure to pulmonary haemorrhage in exercising horses. Equine Vet. J. 32:379-384. 
Magid J.H., Manohar M., Goetz T.E., Baker G.J., Ulbricht R., Bontkowski S. \& Ghantous S. 2000. Pulmonary vascular pressures of Thoroughbred horses exercised 1, 2, 3 and $4 \mathrm{~h}$ after furosemide administration. J. Vet. Pharmacol. Ther. 23:81-89.

Manohar M., Hutchens E. \& Coney E. 1993. Pulmonary haemodynamics in the exercising horse and their relationship to exercise-induced pulmonary haemorrhage. Brit. Vet. J. 149:419-128.

Mills P.C., Marlin D.J. \& Scott C.M. 1996. Pulmonary artery pressure during exercise in the horse after inhibition of nitric oxide synthase. Brit. Vet. J. 152:119-122.

Milne D.W., Muir W.W. \& Skarda R.T. 1975. Pulmonary arterial wedge pressures: blood gas tensions and $\mathrm{pH}$ in the resting horse. Am. J. Vet. Res. 36:1431-1434.

Muylle E., Nuytten J., Deprez P., Van Den Hende C. \& Oyaert W. 1984. Pulmonary driving pressure as a pulmonary function test in horses. J. Equine Vet. Sci. 4:57-59.

Parks C.M. \& Manohar M. 1983. Distribution of blood flow during moderate and strenuous exercise in ponies (Equus caballus). Am. J. Vet. Res. 4:1861-1866.
Poole D.C. \& Erickson H.H. 2004. Heart and vessels: Function during exercise and response to training, p.699-727. In: Hinchcliff K.W., Kaneps A.J. \& Geor R.J. (Eds), Equine Sports Medicine and Surgery. W.B. Saunders, Philadelphia.

Robinson N.E. 1991. The respiratory system, p.7-38. In: Muir W.W. \& Hubbell J.A.E. (Eds), Equine anesthesia: Monitoring and emergency therapy. $2^{\text {nd }}$ ed. Mosby, St Louis.

Rose R.J. \& Hodgson D.R. 1994. An overview of performance and sports medicine, p.5-11. In: Ibid. (Eds), The Athletic Horse. W.B. Saunders, Philadelphia.

Sirotsky C.O., Santiago J.M., Almeida F.Q., Silva L.L.F., Miranda A.C.T., Carrilho S.S. \& Andrade A.M. 2010. Frequência cardíaca de equinos de concurso completo de equitação em teste em esteira de alta velocidade e prova de cross country. Anais XI Conferência Anual da Abraveq, São Paulo, SP, online, (Resumo)

Watanabe M.J., Thomassian A., Teixeira Neto F.J., Alves A.L.G., Hussni C.A. \& Nicoletti J.L.M. 2006. Alterações do pH, da $\mathrm{PO}_{2}$ e da $\mathrm{PCO}_{2}$ arteriais e da concentração de lactato sangüíneo de cavalos da raça Árabe durante exercício em esteira de alta velocidade. Arq. Bras. Med. Vet. Zootec. 58:320-326. 